\title{
CONTRIBUTORS
}

ROSEMARY K. BARRACLOUGH

Disease and Vector Research Group

Institute of Natural Sciences

Massey University, Albany Campus

Private Bag 102-904, NSMC

Auckland, New Zealand

r.k.barraclough@massey.ac.nz

SABIR BIN MUZAFFAR

Department of Biology Faculty of Science

United Arab Emirates University P.O. Box 17551

Al Ain, United Arab Emirates

s_muzaffar@uaeu.ac.ae

DIANNE H. BRUNTON

Ecology \& Conservation Research Group

Institute of Natural Sciences

Massey University, Albany Campus

Private Bag 102-904, NSMC

Auckland, New Zealand

d.h.brunton@massey.ac.nz

DONALD F. CACCAMISE

New Mexico State University

Agricultural Experimental Station

P.O. Box 30003, MSC 3BF

Las Cruces, NM 88003, USA

dcaccami@nmsu.edu

BRUCE K. CAHILL

Vector-borne Disease Laboratory

Maine Medical Center Research Institute

75 John Roberts Road, Suite 9B

South Portland, ME 04106, USA

cahilb@mail.mmc.org
TANEAL M. COPE

Department of Zoology

University of Melbourne

Parkville Campus

Melbourne 3010, Australia

taneal.cop@gmail.com

\section{REBECCA CREAMER}

Department of Entomology

Plant Pathology and Weed Science

New Mexico State University

Skeen Hall Room N141

P.O. Box 30003, MSC 3BE

Las Cruces, NM 88003, USA

creamer@nmsu.edu

ROBERT J. DUSEK

USGS National Wildlife Health Center

6006 Schroeder Road

Madison, WI 53711, USA

rdusek@usgs.gov

JEANNE M. FAIR

Los Alamos National Laboratory

Biosecurity and Public Health

P.O. Box 1663

Los Alamos, NM 87545, USA

jmfair@lanl.gov

ERIK K. HOFMEISTER

USGS Fort Collins Science Center

2150 Centre Avenue, Building C

Fort Collins, CO 80526, USA

ehofmeister@usgs.gov 
WILLIAM M. IKO

USGS National Wildlife Health Center

6006 Schroeder Road

Madison, WI 53711, USA

kob@usgs.gov

IAN L. JONES

Department of Biology

Memorial University of Newfoundland

St. John's, NL A1B 3X9, Canada

iljones@mun.ca

ELEANOR H. LACOMBE

Vector-borne Disease Laboratory

Maine Medical Center Research Institute

75 John Roberts Road, Suite 9B

South Portland, ME 04106, USA

elacombe@midcoast.com

JENNIFER LAVERS

Department of Biology

Memorial University of Newfoundland

St. John's, NL A1B 3X9, Canada

jennifer.lavers@csiro.au

CHARLES B. LUBELCZYK

Vector-borne Disease Laboratory

Maine Medical Center Research Institute

75 John Roberts Road, Suite 9B

South Portland, ME 04106, USA

lubelc@mmc.org

BABETTA L. MARRONE

Los Alamos National Laboratory

Biosecurity and Public Health

P.O. Box 1663

Los Alamos, NM 87545, USA

blm@lanl.gov

ROBERT G. MCLEAN

National Wildlife Research Center

WS/APHIS/USDA

4101 Laporte Avenue

Fort Collins, CO 80521-2154, USA

robert.g.mclean@aphis.usda.gov

PATRICIA G. PARKER

University of Missouri-St.Louis

Whitney R. Harris World Ecology Center

Department of Biology

One University Boulevard

St. Louis, MO 63121, USA

(Current address: Saint Louis Zoo

One Government Drive

St. Louis, MO 63110, USA

pparker@umsl.edu)
ELLEN PAUL

Ornithological Council

5107 Sentinel Drive

Bethesda, MD 20816, USA

ellen.paul@verizon.net

MICHAEL A. PEIRCE

MP International Consultancy

6 Normandale House

Normandale Bexhill-on-Sea

East Sussex, TN39 3NZ, United Kingdom mapeirce.mpic@btinernet.com

\section{A. TOWNSEND PETERSON}

Natural History Museum and Biodiversity

Research Center

University of Kansas

Lawrence, KS 66045, USA

town@ku.edu

ORNITHOLOGICAL COUNCIL

5107 Sentinel Drive

Bethesda, MD 20816, USA

ellen.paul@verizon.net

PETER W. RAND

Vector-borne Disease Laboratory

Maine Medical Center Research Institute

75 John Roberts Road, Suite 9B

South Portland, ME 04106, USA

randp@mmc.org

MARTA REMMENGA

National Surveillance Unit

USDA/APHIS/VS/CEAH

2150 Centre Avenue, Building B,

Mail Stop 2E6

Fort Collins, CO 80526, USA

marta.d.remmenga@aphis.usda.gov

ROBERT E. RICKLEFS

University of Missouri-St. Louis

Whitney R. Harris World Ecology Center

Department of Biology

One University Boulevard

St. Louis, MO 63121, USA

ricklefs@umsl.edu

DIEGO SANTIAGO-ALARCON

University of Missouri-St.Louis

Whitney R. Harris World Ecology Center

Department of Biology

One University Boulevard

St. Louis, MO 63121, USA

onca77@yahoo.com 
YULIN SHOU

Los Alamos National Laboratory

Biosecurity and Public Health

P.O. Box 1663

Los Alamos, NM 87545, USA

shou@lanl.gov

ROBERT P. SMITH, JR.

Vector-borne Disease Laboratory

Maine Medical Center Research Institute

75 John Roberts Road, Suite 9B

South Portland, ME 04106, USA

smithr@mmc.org
KIRSTEN J. TAYLOR-MCCABE

Los Alamos National Laboratory

Biosecurity and Public Health

P.O. Box 1663

Los Alamos, NM 87545, USA

kjmccab@lanl.gov

HOLLY B. VUONG

Department of Fishery and Wildlife Sciences

New Mexico State University

2980 South Espina, Knox Hall 132

P.O. Box 30003, MSC 4901

Las Cruces, NM 88003, USA

hvuong@eden.rutgers.edu 
This page intentionally left blank 